\title{
Strain-Dependent Variability of Early Discovery Small Molecule Pharmacokinetics in Mice: Does Strain Matter?
}

\author{
John T. Barr, Thuy B. Tran, Brooke M. Rock, Jan L. Wahlstrom, and Upendra P. Dahal \\ Pharmacokinetics and Drug Metabolism, Amgen Inc., South San Francisco, California
}

Received January 27, 2020; accepted May 19, 2020

\begin{abstract}
Drug discovery programs routinely perform pharmacokinetic (PK) studies in mice to prioritize lead compounds based on anticipated exposure-efficacy and exposure-toxicity relationships. Because of logistical and/or technical issues, the strain of mouse in early discovery PK studies may not always match the strain in toxicity or efficacy studies. This elicits the question do appreciable straindependent differences in PK parameters exist to an extent that would warrant conducting PK studies in a strain that matches efficacy and toxicity models? To understand the impact that strain may have on PK parameters, we selected eight marketed drugs with well characterized absorption, distribution, metabolism, and excretion properties and diverse structures to perform PK studies in three common mouse strains (Bagg Albino c, C57BL/6, and CD-1). Some statistical strain-dependent differences were observed; however, we found good general agreement of PK parameters between
\end{abstract}

In drug discovery, preclinical pharmacokinetic (PK) studies are critical to the process of optimizing hits to leads and leads to clinical candidates. From an absorption, distribution, metabolism, and excretion (ADME) perspective, PK studies can inform liabilities for parameters such as clearance, volume of distribution, half-life, and bioavailability and may serve as a differentiating factor to prioritizing suitable molecules with optimal ADME properties for pipeline advancement. Furthermore, preclinical PK studies are fundamental to understand the exposure-driven pharmacodynamic (PD) and toxicological effects of a drug candidate.

Preclinical PK studies are routinely performed at an early stage of a discovery program for numerous drug leads, which may result in a high cost and resource burden. To partially mitigate the resource burden, rodent models are typically the first preclinical PK studies conducted; these studies are relatively less expensive and provide rapid measurement of PK parameters. In an ideal case, the rodent strain used for PK studies would match both the PD and toxicology (Tox) model for each drug target of interest, as this would ensure consistency of drug metabolism enzyme and transporter abundances, physiology related to drug excretion and distribution, and thereby bolster confidence in the translation of PD and toxicokinetic exposure-effect models. However, given the logistical challenges (e.g., maintenance of multiple strain

https://doi.org/10.1124/dmd.120.090621. strains: $88 \%, 100 \%, 75 \%, 76 \%, 94 \%$, and $88 \%$ of compounds were within twofold across strains for clearance, volume of distribution at steady state, $t_{1 / 2}, C_{\max }, T_{\max }$, and oral bioavailability, respectively. Overall, we recommend that an approach using a single strain of mouse is appropriate for discovery screening PK studies, provided that proper caution is exercised.

\section{SIGNIFICANCE STATEMENT}

The mouse strain in discovery pharmacokinetic (PK) studies may not match the strain in efficacy and toxicology studies. Currently, there is a gap in the literature addressing whether differences in PK parameters across mouse strains exist such that multiple PK studies are warranted. The results from this study indicated that the PK properties of clinically used drugs between mouse strains are within an acceptable range such that single strain PK is appropriate. colonies, coordinating dosing events, and resources for bioanalytical analysis), early discovery PK is often measured in a single strain that does not necessarily match the PD model and/or the Tox strain. Furthermore, the in vitro liver preparations (microsomes, S9, and hepatocytes) that are used for primary screening are also typically derived from a single strain, most commonly (in our experience) CD-1. With this in mind, we asked the following question specifically as it relates to mice: do empirical strain-dependent differences in $\mathrm{PK}$ parameters exist to the extent that would warrant matching the PK strain with the PD and Tox strain in early drug discovery?

Studies addressing the question of PK variability associated with strain selection are extremely sparse in the scientific literature. MacCallum and Odds (2002) observed a statistically higher exposure in Bagg Albino c (BALB/c) mice relative to DBA/2 for itraconazole. McCarthy et al. (2004) compared the pharmacokinetics of the psychostimulant cocaine in CD-1 and C57BL/6 mice with chronic dosing and reported higher cocaine exposure in C57 black $6(\mathrm{C} 57 \mathrm{BL} / 6)$ mice compared with CD-1 mice. To date, there has not been a controlled systematic evaluation of the effect of mouse strain on PK using a diverse set of compounds in commonly used mouse strains (BALB/c, C57BL/6, and $\mathrm{CD}-1$ ).

In this study, our central hypothesis was that based on similarities of known physiologic parameters, it is not expected that appreciable differences in PK between mouse strains would be found. To test this, we selected eight marketed drugs with well characterized properties and diverse structures (Fig. 1). The compounds were chosen to represent

ABBREVIATIONS: ADME, absorption distribution metabolism and elimination; BALB/c, Bagg Albino c; $C L$, clearance; $\mathrm{CL}_{\text {int }}$, intrinsic clearance; $\mathrm{F}$, oral bioavailability; IS, internal standard; PD, pharmacodynamics; PK, pharmacokinetics; $t_{1 / 2}$, half-life; $T_{\max }$, time to reach $C_{\max } ; T_{0 x}$, toxicology; $V_{\text {ss }}$, volume of distribution at steady state. 


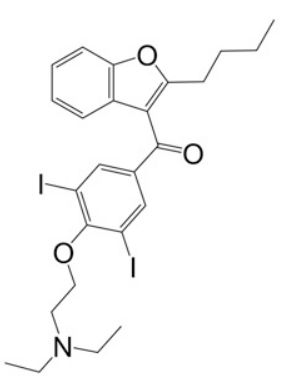

amiodarone<smiles>CC(C)NCC(O)(O)COc1cccc2ccccc12</smiles>

propranolol<smiles>CC1CC2C3CCC4=CC(=O)C=CC4(C)C3C(O)C[C@]2(O)C1C(=O)CO</smiles>

dexamethasone<smiles>CN(C)CCCN1c2ccccc2Sc2ccc(Cl)cc21</smiles>

chlorpromazine
Fig. 1. Chemical structures of compounds used in this study.<smiles>CC1COc2c(N3CCN(C)CC3)c(F)cc3c(=O)c(C(=O)O)cn1c23</smiles>

levofloxacin<smiles>CC(CS)C(=O)N1CCCC1C(=O)O</smiles>

captopril<smiles>COc1ccc2cc(C(C)C(=O)O)ccc2c1</smiles>

naproxen<smiles>CC(C)n1c(/C=C/C(O)CC(O)CC(=O)O)c(-c2ccc(F)cc2)c2ccccc21</smiles>

fluvastatin diverse drug space, accounting for several factors, including molecular weight (200-650 Da), compound class (acid, base, neutral, and zwitterion), lipophilicity ( $\log \mathrm{D}-4.6$ to 5.9$)$, and clearance mechanism (metabolism, unchanged drug excretion, and renal or biliary excretion) as shown in Table 1. We performed both intravenous and oral dosed PK studies in three of the most commonly used strains for preclinical mouse experiments: BALB/c, C57BL/6, and CD-1. Herein, we discuss the implications and estimated risk associated with using a single mouse strain for all PK studies in early drug discovery.

\section{Materials and Methods}

Chemicals and Materials. Eight commercial drugs (Fig. 1) were selected to represent diverse drug space and clearance mechanisms in humans (Table 1). Test compounds (amiodarone, captopril, chlorpromazine, dexamethasone, fluvastatin,

TABLE 1

Physicochemical properties of compounds and primary elimination route in humans

\begin{tabular}{|c|c|c|c|c|c|}
\hline Compound & MW & Class & $\mathrm{pKa}^{a}$ & $\log \mathrm{D}($ at $\mathrm{pH} 7.4)$ & Primary Route of Elimination \\
\hline Amiodarone & 645.3 & Acidic & 6.6 & 5.9 & Hepatic metabolism (CYP3A and 2C8) and biliary excretion of metabolites ${ }^{b}$ \\
\hline Captopril & 217.3 & Zwitterion & $3.7,9.8$ & -2.8 & Urinary excretion of unchanged drug $(40 \%-50 \%)+$ disulfide dimer or cysteine conjugate ${ }^{c}$ \\
\hline Chlorpromazine & 318.9 & Basic & 9.3 & 3.2 & Metabolism (oxidative) and urinary excretion of metabolites $(43 \%-65 \%)^{d}$ \\
\hline Dexamethasone & 392.5 & Neutral & - & -4.6 & Hepatic metabolism (CYP3A) and urinary excretion of metabolites $(65 \%)^{e}$ \\
\hline Fluvastatin & 411.5 & Acidic & 4.5 & -2.2 & Hepatic metabolism $\left(\right.$ CYP2C9) and biliary elimination $(\sim 90 \%)^{f}$ \\
\hline Levofloxacin & 361.4 & Zwitterion & $6.2,8.7$ & -0.39 & Urinary excretion of unchanged drug $(\sim 87 \%)^{g}$ \\
\hline Naproxen & 230.3 & Acidic & 4.2 & 0.35 & Urinary excretion of glucuronide metabolite $(66 \%-92 \%)^{h}$ \\
\hline Propranolol & 259.4 & Basic & 9.4 & 0.79 & Metabolism (oxidative - CYP1A2 and 2D6 and glucuronidation) ${ }^{i}$ \\
\hline
\end{tabular}

${ }^{a}$ https://pubchem.ncbi.nlm.nih.gov.

${ }^{b}$ https://www.accessdata.fda.gov/drugsatfda_docs/label/2015/018972s047lbl.pdf.

${ }^{c}$ https://www.accessdata.fda.gov/drugsatfda_docs/label/2012/018343s084lbl.pdf.

${ }^{d}$ https://genesight.com/wp-content/uploads/2017/05/Chlorpromazine-Thorazine-FDA-Label.pdf.

${ }^{e}$ https://www.medsafe.govt.nz/profs/Datasheet/d/DblDexamethasoneNewFormulationinj.pdf.

https://www.accessdata.fda.gov/drugsatfda_docs/label/2007/020261s039,021192s013lbl.pdf.

${ }^{g} \mathrm{https} / / /$ www.accessdata.fda.gov/drugsatfda_docs/label/2007/020634s045,020635s048,021721s031lbl.pdf.

${ }^{h} \mathrm{https}: / / \mathrm{www}$.accessdata.fda.gov/drugsatfda_docs/label/2011/020353s028lbl.pdf.

${ }^{i} \mathrm{https}: / /$ www.accessdata.fda.gov/drugsatfda_docs/label/2011/016418s080,016762s017,017683s008lbl.pdf. 


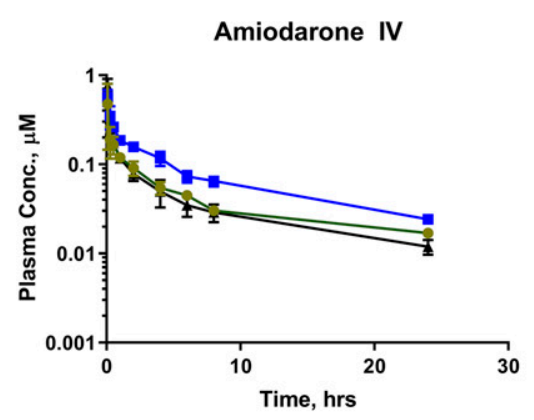

Dexamethasone IV

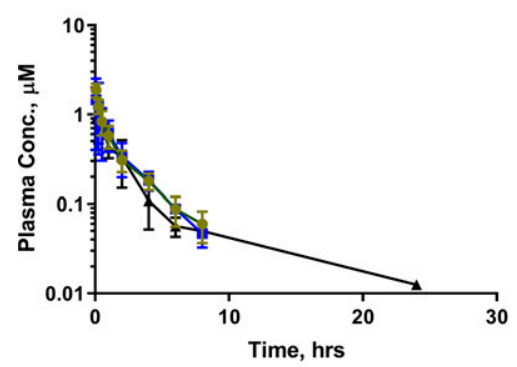

Naproxen IV

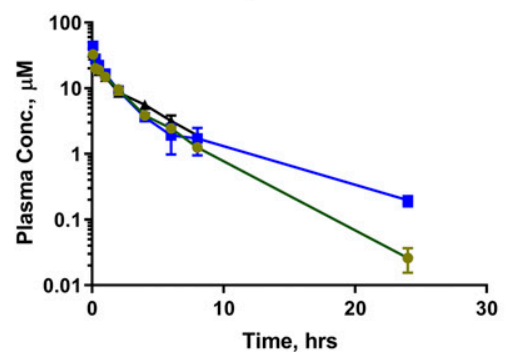

Captopril IV

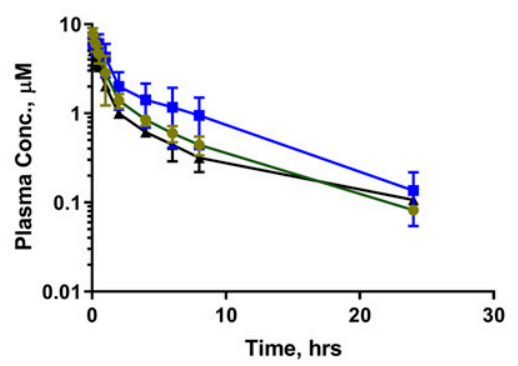

Fluvastatin IV

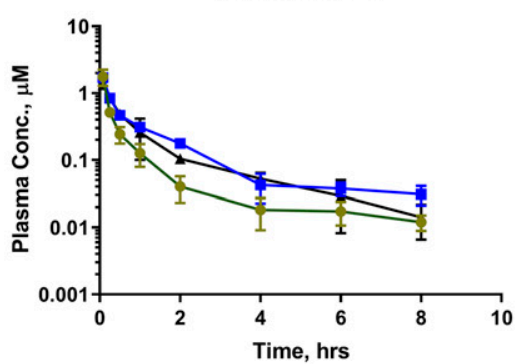

Propanolol IV

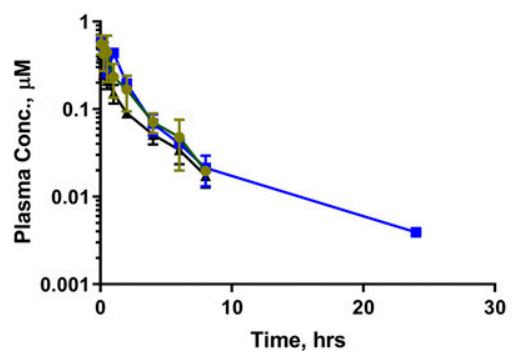

Chlorpromazine IV

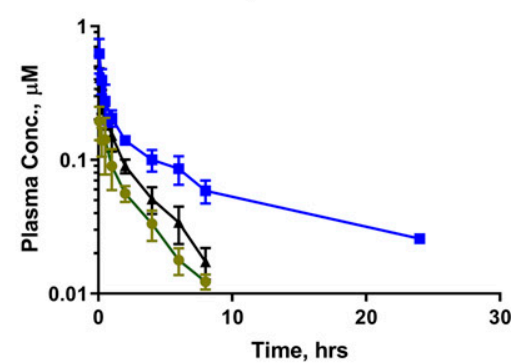

Levofloxacin IV

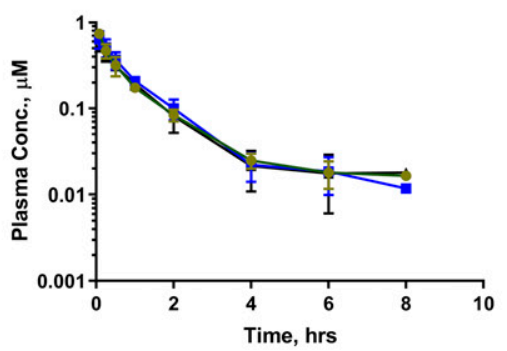

Fig. 2. Plasma concentration-time profiles of compounds in CD1, C57BL/6, and BALB/c mice after a $1 \mathrm{mg} / \mathrm{kg}$ i.v. dose (black triangle, CD-1; blue square, C57BL/6; and green circle, BALB/c). Markers and error bars represent the mean and S.D., respectively, for three subjects.

levofloxacin, naproxen, and propranolol) for in vitro clearance experiments were obtained as a 10-mM solution in DMSO from Selleck Chemicals (Houston, TX). Suspended CD-1 (male, pooled, $n=18$, lot BVU), C57BL/6 (male, pooled, $n=$ 50 , lot BSE), and BALB/c (male, pooled, $n=45$, lot CYG) primary hepatocytes and INVITROGRO HT media were obtained from Bioreclamation IVT (Baltimore, MD). Compound solids (amiodarone, captopril, chlorpromazine, dexamethasone, fluvastatin, levofloxacin, naproxen, and propranolol) for in vivo testing were obtained from Amgen's internal compound library. DMSO, acetonitrile, hydroxypropyl methylcellulose, and formic acid were obtained from Sigma Aldrich (St. Louis, MO). Dulbecco's modified eagle medium was purchased from Gibco (Dublin, Ireland). All other reagents were analytical quality or better. Male CD-1, C57BL/6, and BALB/c mice were obtained from Envigo (Huntingdon, UK).

Measurement of Pharmacokinetic Parameters in Mice. Mice were housed in groups at an Association for Assessment and Accreditation of Laboratory Animal Care International-accredited facility. Animals were cared for in accordance with the Guide for the Care and Use of Laboratory Animals, 8th Edition. All research protocols were reviewed and approved by the Amgen Institutional Animal Care and Use Committee. Mice (CD-1, C57BL/6, and BALB/c; Envigo, US/Netherlands; 8-12 weeks old; male) were housed in individual ventilated caging system on an irradiated corncob bedding (Teklad 7097; Envigo). Lighting in animal holding rooms was maintained on a 12-hour light/dark cycle, and the ambient temperature and humidity range was at $68-79^{\circ} \mathrm{F}$ and $30 \%-70 \%$, respectively. Animals had ad libitum access to irradiated pelleted feed (Teklad Global Rodent Diet, soy protein free extruded 2020X; Envigo) and reverse-osmosis-chlorinated (0.3-0.5 ppm) water via an automatic watering system. Cages were changed biweekly inside an engineered cage changing station. For intravenous dosing, compounds were formulated in DMSO at a concentration of $2 \mathrm{mg} / \mathrm{ml}$. Using the appropriate volume, compounds were administered intravenously at a final dose of $1 \mathrm{mg} / \mathrm{kg}$ via femoral vein cannula. For oral dosing, compounds were formulated in $1 \%$ Tween $80,2 \%$ hydroxypropyl methylcellulose, and $97 \%$ water at a concentration of $0.5 \mathrm{mg} / \mathrm{ml}$. The oral formulations were solution, except for dexamethasone, which had suspension formulation. Using the appropriate volume, compounds were administered by mouth at a final dose of $5 \mathrm{mg} / \mathrm{kg}$. Compounds were dosed to three animals per group. Blood was collected at 0.083 (intravenously only), 0.25, 0.5, 1, 2, 4, 6, 8, and 24 hours after dosing. At each time point, approximately $20 \mu \mathrm{l}$ of blood was collected serially through saphenous vein (alternatively from each leg for consecutive time points) from each subject. Samples were collected in $\mathrm{K}_{2}$ EDTA tubes and placed on wet ice until centrifuged $\left(\right.$ at $4{ }^{\circ} \mathrm{C}, 10$ minutes at $13,000 \mathrm{rpm}$, within 1 hour of collection) to separate plasma. Plasma samples were separated into polypropylene tubes and 96-well deep well plates and were transferred immediately after separation to freezer $\left(-70^{\circ} \mathrm{C}\right)$ up to 24 hours (or until analysis). Plasma concentrations were determined by using the calibration curves prepared in plasma matrix for each analyte; briefly, $10 \mu \mathrm{l}$ of calibration standards, blank, blank + internal standard (IS), and study samples were transferred to a $0.6-\mathrm{ml} 96$-well deep well plate. One hundred fifty microliters of IS solution $(0.1 \mu \mathrm{g} / \mathrm{ml}$ verapamil in acetonitrile) 


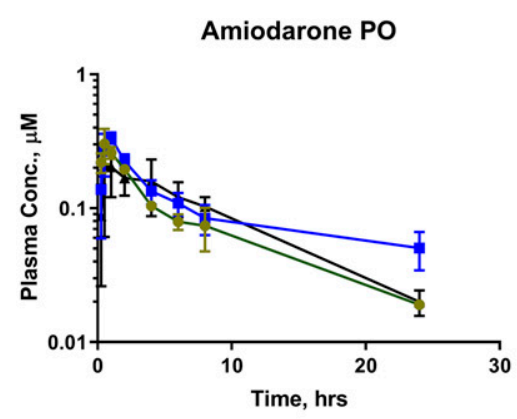

Dexamethasone PO

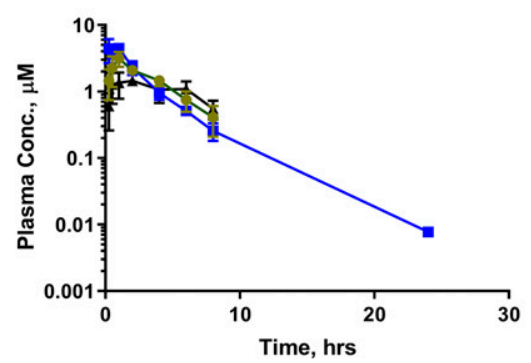

Naproxen PO

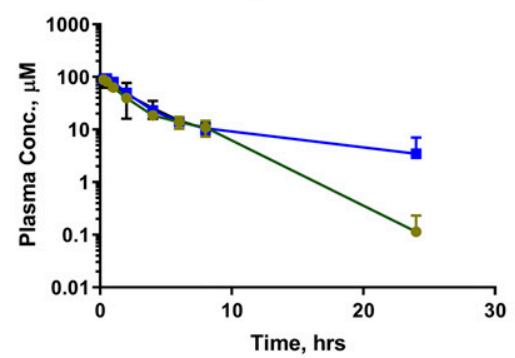

Captopril PO

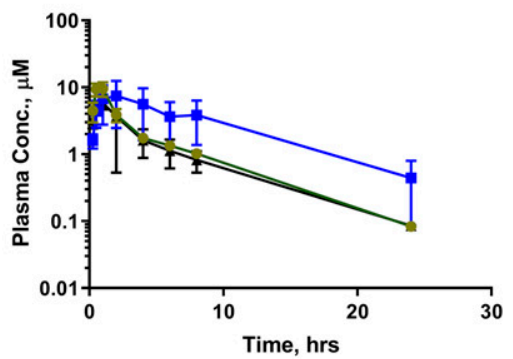

Fluvastatin PO

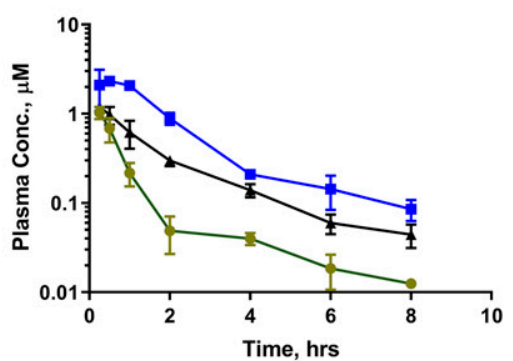

Propanolol PO

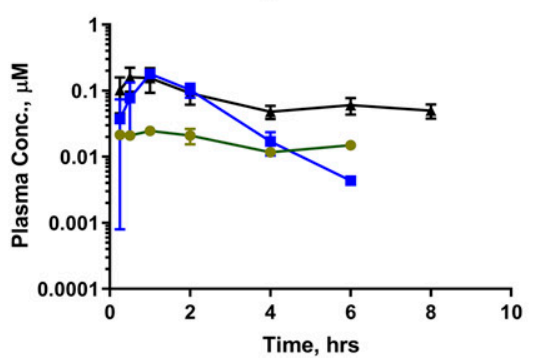

Chlorpromazine PO

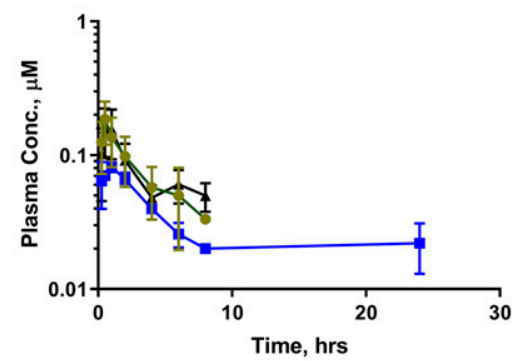

Levofloxacin PO

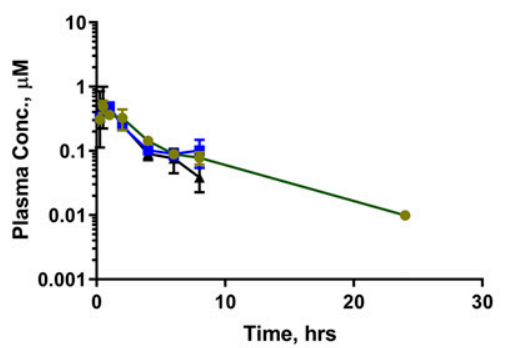

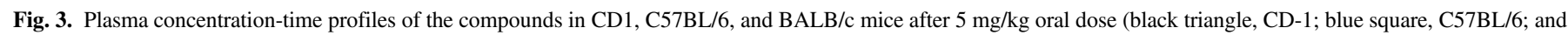
green circle, BALB/c). Markers and error bars represent the mean and S.D., respectively, for three subjects.

was added to all the samples except for the two blank samples. One hundred fifty microliters of acetonitrile was added to both of the blank samples. All samples were vortexed for 10 minutes and centrifuged at $3200 \mathrm{~g}$ for 10 minutes. Approximately $125 \mu l$ of the supernatant was transferred to a 96-well deep well plate. Analytes were measured by using liquid chromatography-tandem mass spectrometry as described previously (Barr et al., 2019). Pharmacokinetic parameters were obtained for each individual animal via noncompartmental analysis of the data in Watson LIMS 7.5
(Thermo Fisher Scientific). Individuals that had greater than 20\% extrapolation of area under the curve were excluded from the analysis.

Determination of Clearance in Primary Suspended Mouse Hepatocytes. Test compounds were obtained as a $10-\mathrm{mM}$ stock in DMSO and subsequently diluted to a working solution of $50 \mu \mathrm{M}$ in 1:1 acetonitrile-water. Cryopreserved hepatocytes were thawed at $37^{\circ} \mathrm{C}$ and gently rinsed by inversion with $50 \mathrm{ml}$ of invitroGRO HT media. The cell suspension was centrifuged at room temperature for 3 minutes at $65 \mathrm{~g}$. The resultant supernatant was discarded, and cells were

TABLE 2

Measured CL parameters and the interstrain comparisons for eight compounds

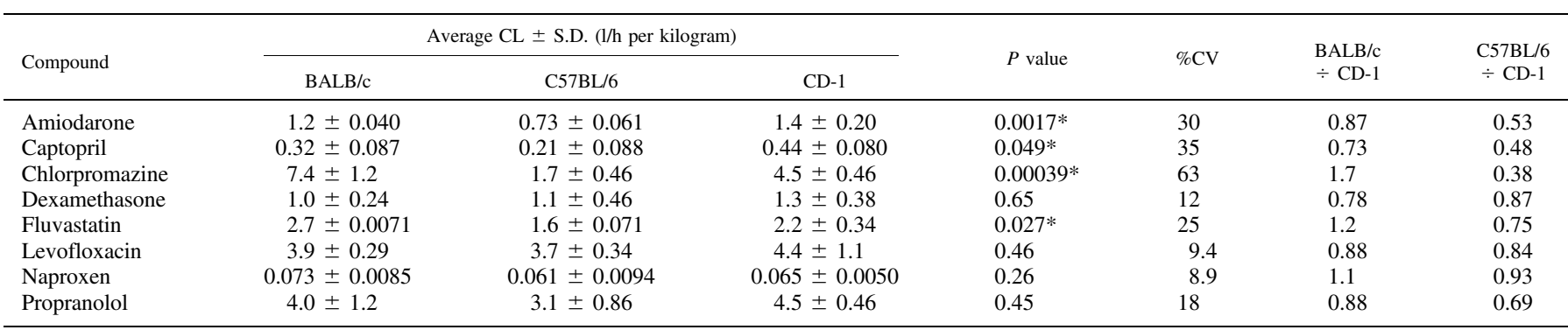

${ }^{*} P$ value of 0.05 or less found by a one-way ANOVA test. 
TABLE 3

Measured $\mathrm{V}_{\mathrm{ss}}$ parameters and the interstrain comparisons for eight compounds

\begin{tabular}{|c|c|c|c|c|c|c|c|}
\hline \multirow{2}{*}{ Compound } & \multicolumn{3}{|c|}{ Average $V_{\mathrm{ss}} \pm$ S.D. $(1 / \mathrm{kg})$} & \multirow{2}{*}{$P$ value } & \multirow{2}{*}{$\% \mathrm{CV}$} & \multirow{2}{*}{$\begin{array}{c}\text { BALB/c } \\
\div \mathrm{CD}-1\end{array}$} & \multirow{2}{*}{$\begin{array}{l}\mathrm{C} 57 \mathrm{BL} / \mathrm{C} \\
\div \mathrm{CD}-1\end{array}$} \\
\hline & $\mathrm{BALB} / \mathrm{c}$ & C57BL/6 & CD-1 & & & & \\
\hline Amiodarone & $18 \pm 2.8$ & $10 \pm 0.45$ & $15 \pm 4.4$ & $0.036^{*}$ & 29 & 1.2 & 0.67 \\
\hline Captopril & $1.6 \pm 0.020$ & $1.6 \pm 0.68$ & $2.2 \pm 0.78$ & 0.42 & 20 & 0.72 & 0.72 \\
\hline Chlorpromazine & $27 \pm 4.7$ & $18 \pm 3.0$ & $15 \pm 4.9$ & $0.031 *$ & 32 & 1.8 & 1.2 \\
\hline Dexamethasone & $2.8 \pm 0.42$ & $4.1 \pm 3.7$ & $4.6 \pm 2.1$ & 0.69 & 24 & 0.62 & 0.89 \\
\hline Fluvastatin & $4.1 \pm 1.1$ & $5.0 \pm 1.4$ & $4.1 \pm 1.3$ & 0.71 & 12 & 1.0 & 1.2 \\
\hline Levofloxacin & $8.3 \pm 3.8$ & $7.4 \pm 1.9$ & $8.5 \pm 2.0$ & 0.87 & 7.4 & 0.97 & 0.87 \\
\hline Naproxen & $0.22 \pm 0.024$ & $0.26 \pm 0.028$ & $0.22 \pm 0.024$ & 0.14 & 10 & 0.97 & 1.2 \\
\hline Propranolol & $9.4 \pm 2.4$ & $11 \pm 2.4$ & $6.2 \pm 5.1$ & 0.12 & 27 & 1.5 & 1.7 \\
\hline
\end{tabular}

${ }^{*} P$ value of 0.05 or less found by a one-way ANOVA test.

resuspended in Dulbecco's modified eagle medium media (prewarmed to $37^{\circ} \mathrm{C}$ ) to have a final density of 0.5 million live cells $/ \mathrm{ml}$. Eight hundred microliters of the cell suspension was dispensed into wells in a 96-well plate. Incubations were initiated by addition of $8 \mu \mathrm{l}$ of compound working solution described above, resulting in a final concentration of $0.5 \mu \mathrm{M}$ substrate with $0.5 \% \mathrm{v} / \mathrm{v}$ acetonitrile. At $0,10,30,60,90$, and 120 minutes, reactions were stopped by addition of $300 \mu \mathrm{l}$ of acetonitrile containing $0.1 \% \mathrm{v} / \mathrm{v}$ formic acid and $1 \mu \mathrm{M}$ tolbutamide as IS. Upon incubation termination, plate wells were capped to avoid evaporation. Upon completion of all time points, sample plates were vortexed at room temperature for 10 minutes and subsequently centrifuged at $3220 \mathrm{~g}$ for 20 minutes at $4^{\circ} \mathrm{C}$. Supernatants were transferred to a separate plate for analysis. Analytes were measured by using liquid chromatography-tandem mass spectrometry as described previously (Barr et al., 2019). Incubations were performed in duplicate for each analyte. In vitro intrinsic clearance $\left(\mathrm{CL}_{\text {int }}\right)$ was calculated from the parent compound depletion in each incubation (eq. 1) as follows:

$$
\begin{aligned}
C L_{\text {int }}= & - \text { slope of } \ln (\% \text { drug remaining }) \text { vs time } \\
& \times \frac{\text { mL incubation }}{\text { million cells }} \mu \mathrm{l} \cdot \mathrm{min}^{-1} \cdot \text { mil cells }{ }^{-1} .
\end{aligned}
$$

Statistical Analyses. To test for statistical differences in parameters measured between different mouse strains, a single factor ANOVA test was performed in Microsoft Excel. A $P$ value of 0.05 or less was used as a threshold for statistical significance. Groups without enough data for a proper ANOVA analysis were denoted with a $P$ value of not determined

\section{Results}

Compounds Were Selected for Diversity. All eight test compounds are marketed drugs with well characterized properties and diverse structures (Fig. 1). The compounds were selected to represent diverse drug space, accounting for several factors including molecular weight, compound class, lipophilicity, and clearance mechanism in humans (Table 1). Overall, the physicochemical properties were represented as follows: molecular weight varied from approximately 200-650 Da, all charge states (acidic, basic, neutral, and zwitterionic) were represented, and $\log \mathrm{D}$ ranged from low to high lipophilicity ( -4.6 to 5.9$)$. Multiple primary clearance mechanisms were represented by the group, including urinary excretion, biliary excretion, phase I metabolism by various cytochrome P450s (CYP1A2, 2C8, 2C9, 2D6, and 3A), and phase II metabolism by UDP-glucuronosyltransferases.

Measurement and Cross-Strain Comparison of PK in Mice. Concentration versus time profiles for compounds dosed intravenously and orally are shown in Figs. 2 and 3, respectively. Intravenous clearance (CL), volume of distribution at steady state $\left(\mathrm{V}_{\mathrm{ss}}\right)$, half-life $\left(t_{1 / 2}\right)$, and oral bioavailability $(\mathrm{F})$ were all determined by noncompartmental analysis. $C_{\max }$ and time to reach $C_{\max }\left(T_{\max }\right)$ were based on observed plasma concentration time profile. For each parameter, the data across three mouse strains were compared in multiple ways. First, a single-factor ANOVA was performed to assess the statistical significance of differences between groups; in this way, the analysis is used to test the null hypothesis that the average value of the observed parameter is the same for all strains. For the purposes of this analysis, a $P$ value less than or equal to 0.05 was used as the threshold for statistical significance. Second, the coefficient of variation was also calculated as a measure of the data dispersion between strains. Third, a simple fold comparison was made, using CD-1 as the comparator strain; this was calculated by taking the measured parameter in each strain (either $\mathrm{C} 57 \mathrm{BL} / 6$ or $\mathrm{BALB} / \mathrm{c}$ ) and dividing it by the corresponding value in CD-1.

Clearance values for different test compounds ranged from 0.061 to 7.4 1/h per kilogram. Using an ANOVA analysis of CL, a $P$ value of less than 0.05 was not observed for $50 \%$ of compounds. Percent $C V$ between strains was less than $50 \%$ for $88 \%$ of compounds. Clearance for BALB/c relative to CD-1 was within twofold for $100 \%$ of compounds tested, and

TABLE 4

\begin{tabular}{|c|c|c|c|c|c|c|c|}
\hline \multirow{2}{*}{ Compound } & \multicolumn{3}{|c|}{ Average $t_{1 / 2}(\mathrm{~h})$} & \multirow{2}{*}{$P$ value } & \multirow{2}{*}{$\% \mathrm{CV}$} & \multirow{2}{*}{$\begin{array}{l}\text { BALB/c } \\
\div \text { CD-1 }\end{array}$} & \multirow{2}{*}{$\begin{array}{c}\mathrm{C} 57 \mathrm{BL} / 6 \\
\div \mathrm{CD}-1\end{array}$} \\
\hline & $\mathrm{BALB} / \mathrm{c}$ & C57BL/6 & CD-1 & & & & \\
\hline Amiodarone & $12 \pm 2.1$ & $11 \pm 0.30$ & $9.9 \pm 1.3$ & 0.46 & 8.5 & 1.2 & 1.1 \\
\hline Captopril & $5.2 \pm 1.9$ & $6.5 \pm 2.6$ & $4.9 \pm 2.4$ & 0.68 & 15 & 1.0 & 1.3 \\
\hline Chlorpromazine & $3.1 \pm 1.1$ & $9.1 \pm 3.4$ & $2.8 \pm 1.1$ & $0.020^{*}$ & 71 & 1.1 & 3.2 \\
\hline Dexamethasone & $2.5 \pm 0.39$ & $2.3 \pm 1.1$ & $4.0 \pm 3.3$ & 0.56 & 32 & 0.62 & 0.57 \\
\hline Fluvastatin & $2.8 \pm 0.67$ & $4.5 \pm 0.40$ & $2.2 \pm 0.70$ & $0.039 *$ & 38 & 1.3 & 2.1 \\
\hline Levofloxacin & $2.7 \pm 1.7$ & $2.6 \pm 1.9$ & $2.5 \pm 1.7$ & 1.0 & 2.5 & 1.1 & 1.0 \\
\hline Naproxen & $2.8 \pm 0.21$ & $5.7 \pm 1.1$ & $2.6 \pm 0.44$ & $0.0030^{*}$ & 47 & 1.1 & 2.2 \\
\hline Propranolol & $1.9 \pm 0.47$ & $4.4 \pm 2.2$ & $1.4 \pm 0.85$ & 0.079 & 63 & 1.3 & 3.2 \\
\hline
\end{tabular}

Measured $t_{1 / 2}$ parameters and the interstrain comparisons for eight compounds

${ }^{*} P$ value of 0.05 or less found by a one-way ANOVA test. 
TABLE 5

Measured F parameters and the interstrain comparisons for eight compounds

\begin{tabular}{|c|c|c|c|c|c|c|c|}
\hline \multirow{2}{*}{ Compound } & \multicolumn{3}{|c|}{ Average F (\%) } & \multirow{2}{*}{$P$ value } & \multirow{2}{*}{$\% \mathrm{CV}$} & \multirow{2}{*}{$\mathrm{BALB} / \mathrm{c} \div \mathrm{CD}-1$} & \multirow{2}{*}{$\begin{array}{c}\text { C57BL/6 } \\
\div \text { CD }-1\end{array}$} \\
\hline & $\mathrm{BALB} / \mathrm{c}$ & C57BL/6 & CD-1 & & & & \\
\hline Amiodarone & $31 \pm 2.3$ & $22 \pm 2.0$ & $38 \pm 8.8$ & $0.028^{*}$ & 27 & 0.80 & 0.57 \\
\hline Captopril & $40 \pm 5.5$ & $57 \pm 37$ & $45 \pm 18$ & 0.69 & 18 & 0.90 & 1.3 \\
\hline Chlorpromazine & $25 \pm 13$ & $7.1 \pm 1.1$ & $17 \pm 1.1$ & 0.065 & 53 & 1.5 & 0.44 \\
\hline Dexamethasone & $99 \pm 12$ & $107 \pm 4.4$ & $99 \pm 15$ & 0.64 & 4 & 1.0 & 1.1 \\
\hline Fluvastatin & $19 \pm 1.7$ & $70 \pm 2.8$ & $37 \pm 4.7$ & $4.0 \mathrm{E}-06^{*}$ & 61 & 0.52 & 1.9 \\
\hline Levofloxacin & $57 \pm 9.2$ & $38 \pm 4.0$ & $48 \pm 3.0$ & $0.022 *$ & 20 & 1.2 & 0.80 \\
\hline Naproxen & $89 \pm 11$ & $99 \pm 6.4$ & $92 \pm 26$ & 0.84 & 5 & 0.97 & 1.1 \\
\hline Propranolol & $1.2 \pm 0.61$ & $5.2 \pm 0.19$ & $6.9 \pm 4.0$ & 0.061 & 65 & 0.19 & 0.74 \\
\hline
\end{tabular}

${ }^{*} P$ value of 0.05 or less found by a one-way ANOVA test.

clearance for C57BL/6 relative to CD-1 was within twofold for $75 \%$ of compounds tested. Overall, clearance for either BALB/c or C57BL6 was within twofold of CD-1 in $88 \%$ of the cases. A detailed summary of the CL data across multiple mouse strains is provided in Table 2.

Volume of distribution values for different test compounds ranged from 0.22 to $27 \mathrm{l} / \mathrm{kg}$. Using an ANOVA of $\mathrm{V}_{\mathrm{ss}}$, a $P$ value of less than 0.05 was not observed for $75 \%$ of compounds. Percent CV between strains was less than $50 \%$ for $100 \%$ of compounds. $V_{\text {ss }}$ for BALB/c relative to $\mathrm{CD}-1$ was within twofold for $100 \%$ of compounds tested, and $\mathrm{V}_{\mathrm{ss}}$ for C57BL/6 relative to CD-1 was within twofold for $100 \%$ of compounds tested. Overall, $\mathrm{V}_{\mathrm{ss}}$ for either BALB/c or C57BL6 was within twofold of CD-1 in 100\% of the cases. A detailed summary of the $\mathrm{V}_{\mathrm{ss}}$ data across multiple mouse strains is provided in Table 3 .

Half-life values for different test compounds ranged from 1.4 to 12 hours. Using an ANOVA of $t_{1 / 2}$, a $P$ value of less than 0.05 was not observed for $63 \%$ of compounds. Percent CV between strains was less than $50 \%$ for $75 \%$ of compounds. Half-life for BALB/c relative to CD-1 was within twofold for $100 \%$ of compounds tested, and half-life for C57BL/6 relative to CD-1 was within twofold for 50\% of compounds tested. Overall, half-life for either BALB/c or C57BL6 was within twofold of CD-1 in $75 \%$ of the cases. A detailed summary of the half-life data across multiple mouse strains is provided in Table 4.

Oral bioavailability values for different test compounds ranged from $1.3 \%$ to $110 \%$. Using an ANOVA of $\mathrm{F}$, a $P$ value of less than 0.05 was not observed for $63 \%$ of compounds. Percent CV between strains was less than $50 \%$ for $63 \%$ of compounds. F for BALB/c relative to CD-1 was within twofold for $88 \%$ of compounds tested, and F for C57BL/6 relative to $\mathrm{CD}-1$ was within twofold for $88 \%$ of compounds tested. Overall, F for either BALB/c or C57BL6 was within twofold of CD-1 in $88 \%$ of the cases. A detailed summary of the $\mathrm{F}$ data across multiple mouse strains is provided in Table 5 .
$C_{\max }$ values for different test compounds ranged from 0.024 to 9.6 $\mu \mathrm{M}$. Using an ANOVA analysis of $C_{\max }$, a $P$ value of less than 0.05 was not observed for $63 \%$ of compounds. Percent $\mathrm{CV}$ between strains was less than $50 \%$ for $63 \%$ of compounds. $C_{\max }$ for $\mathrm{BALB} / \mathrm{c}$ relative to CD- 1 was within twofold for $88 \%$ of compounds tested, and $C_{\max }$ for C57BL/6 relative to CD-1 was within twofold for $63 \%$ of compounds tested. Overall, $C_{\max }$ for either BALB/c or C57BL6 was within twofold of CD1 in $76 \%$ of the cases. A detailed summary of the $C_{\max }$ data across multiple mouse strains is provided in Table 6 .

$T_{\max }$ values for different test compounds ranged from 0.25 to 2 hours. Because $T_{\max }$ is a categorical variable, the data are not suitable for statistical analysis, and therefore ANOVA analysis or CV calculations were not performed. $T_{\max }$ for BALB/c relative to $\mathrm{CD}-1$ was within twofold for $100 \%$ of compounds tested, and $T_{\max }$ for C57BL/6 relative to CD-1 was within twofold for $88 \%$ of compounds tested. Overall, $T_{\max }$ for either BALB/c or C57BL6 was within twofold of CD-1 in $94 \%$ of the cases. A detailed summary of the $T_{\max }$ data across multiple mouse strains is provided in Table 7 .

Assessment of Interday Variability of PK Parameters. For determining typical interday experimental variability, chlorpromazine was selected as an exemplar compound. PK was assessed by using different animal cohorts across 3 days in both CD-1 and C57BL/6 (Table 8). Overall, an ANOVA of $\mathrm{CL}, \mathrm{V}_{\mathrm{ss}}$, half-life, $C_{\max }$, and $\mathrm{F}$ afforded $P$ values greater than 0.05 across interday studies in both CD-1 and $\mathrm{C} 57 \mathrm{BL} / 6$ strains. Interday $\mathrm{CV}$ for these parameters ranged from $8.2 \%$ to $27 \%$.

In Vitro Hepatocyte Clearance. Hepatocyte $\mathrm{CL}_{\text {int }}$ ranged from $<2.5$ to $310 \mu \mathrm{l} / \mathrm{min}$ per million cells. Using an ANOVA of $t_{1 / 2}$, a $P$ value of less than 0.05 was not observed for $88 \%$ of compounds. Percent CV between strains was less than $50 \%$ for $88 \%$ of compounds. $\mathrm{CL}_{\mathrm{int}}$ for $\mathrm{BALB} / \mathrm{c}$ relative to CD-1 was within twofold for $88 \%$ of compounds

TABLE 6

Measured $C_{\max }$ parameters and the interstrain comparisons for eight compounds

\begin{tabular}{|c|c|c|c|c|c|c|c|}
\hline \multirow{2}{*}{ Compound } & \multicolumn{3}{|c|}{ Average $C_{\max }(\mu \mathrm{M})$} & \multirow{2}{*}{$P$ value } & \multirow{2}{*}{$\% \mathrm{CV}$} & \multirow{2}{*}{$\begin{array}{l}\text { BALB/c } \\
\div \text { CD-1 }\end{array}$} & \multirow{2}{*}{$\begin{array}{c}\text { C57BL/6 } \\
\div \text { CD-1 }\end{array}$} \\
\hline & $\mathrm{BALB} / \mathrm{c}$ & C57BL/6 & $\mathrm{CD}-1$ & & & & \\
\hline Amiodarone & $0.32 \pm 0.078$ & $0.33 \pm 0.033$ & $0.24 \pm 0.11$ & 0.38 & 17 & 1.3 & 1.4 \\
\hline Captopril & $9.6 \pm 2.2$ & $7.6 \pm 4.7$ & $6.1 \pm 1.2$ & 0.42 & 23 & 1.6 & 1.2 \\
\hline Chlorpromazine & $0.19 \pm 0.066$ & $0.082 \pm 0.0072$ & $0.17 \pm 0.056$ & 0.095 & 39 & 1.1 & 0.48 \\
\hline Dexamethasone & $3.1 \pm 0.79$ & $5.0 \pm 0.84$ & $1.7 \pm 0.28$ & $0.0028 *$ & 51 & 1.8 & 2.9 \\
\hline Fluvastatin & $1.0 \pm 0.16$ & $2.6 \pm 0.51$ & $1.2 \pm 0.16$ & $0.0020 *$ & 54 & 0.83 & 2.2 \\
\hline Levofloxacin & $0.49 \pm 0.11$ & $0.49 \pm 0.051$ & $0.67 \pm 0.3$ & 0.46 & 19 & 0.73 & 0.73 \\
\hline Naproxen & $88 \pm 7.6$ & $97 \pm 15$ & $90 \pm 13$ & 0.68 & 5.2 & 1.0 & 1.1 \\
\hline Propranolol & $0.024 \pm 0.0043$ & $0.18 \pm 0.0093$ & $0.13 \pm 0.084$ & $0.021 *$ & 72 & 0.18 & 1.4 \\
\hline
\end{tabular}

${ }^{*} P$ value of 0.05 or less found by a one-way ANOVA test. 
TABLE 7

Measured $T_{\max }$ parameters and the interstrain comparisons for eight compounds

\begin{tabular}{|c|c|c|c|c|c|c|}
\hline \multirow{2}{*}{ Compound } & \multicolumn{3}{|c|}{ Average $T_{\max }(\mathrm{h})$} & \multirow{2}{*}{$\% \mathrm{CV}$} & \multirow{2}{*}{$\mathrm{BALB} / \mathrm{c} \div \mathrm{CD}-1$} & \multirow{2}{*}{$\begin{array}{l}\mathrm{C} 57 \mathrm{BL} / 6 \\
\div \mathrm{CD}-1\end{array}$} \\
\hline & $\mathrm{BALB} / \mathrm{c}$ & C57BL/6 & CD-1 & & & \\
\hline Amiodarone & $1(0.5-1)$ & $1(0.5-1)$ & $2(0.5-4)$ & 43 & 0.50 & 0.50 \\
\hline Captopril & $0.5(0.5-1)$ & $2(0.5-2)$ & $0.5(0.5-2)$ & 87 & 1.0 & 4.0 \\
\hline Chlorpromazine & $0.5(0.5-0.5)$ & $1(1-1)$ & $0.5(0.5-1)$ & 43 & 1.0 & 2.00 \\
\hline Dexamethasone & $1(1-1)$ & $0.25(0.25-0.5)$ & $1(0.5-6)$ & 58 & 1.0 & 0.3 \\
\hline Fluvastatin & $0.25(0.25-0.25)$ & $0.5(0.25-0.5)$ & $0.25(0.25-0.25)$ & 43 & 1.0 & 2.0 \\
\hline Levofloxacin & $0.5(0.5-0.5)$ & $1(1-1)$ & $0.5(0.5-1)$ & 43 & 1.0 & 2.0 \\
\hline Naproxen & $0.25(0.25-0.25)$ & $0.5(0.25-0.5)$ & $0.25(0.25-0.5)$ & 43 & 1.0 & \\
\hline Propranolol & $1(0.5-2)$ & $1(1-1)$ & $1(0.5-1)$ & 0 & 1.0 & 1.0 \\
\hline
\end{tabular}

tested, and $\mathrm{CL}_{\text {int }}$ for $\mathrm{C} 57 \mathrm{BL} / 6$ relative to $\mathrm{CD}-1$ was within twofold for $88 \%$ of compounds tested. Overall, $\mathrm{CL}_{\text {int }}$ for either BALB/c or C57BL6 was within twofold of CD-1 in $88 \%$ of the cases. A detailed summary of the $\mathrm{CL}_{\text {int }}$ data across multiple mouse strains is provided in Table 9.

\section{Discussion}

Rodent PK screening is a paramount tool in early small-molecule drug discovery that is used for two primary reasons: 1) to understand ADMErelated liabilities for molecules and 2) to understand the exposureefficacy and exposure-toxicity relationships. Because of efforts (such as a sustainable approach to reduce, replace, and recycle resources) to streamline preclinical PK workflows or resource constraints, it is often the case that the strain in a screening PK experiment may not match the strain in a PD or Tox study. This risk is particularly prevalent in mouse studies, in which the selection of PD model strain is often quite variable, depending largely on the therapeutic area of study, and may even change over the course of a discovery program. Currently, we have identified a gap in the available body of literature: there are no publications addressing the issue of whether an appreciable strain-dependent PK difference exists in mice. To address this, we designed a study using a panel of diverse commercial drugs and systematically evaluated the intravenous and oral PK in BALB/c, C57BL/6, and CD-1 mice. To our knowledge, this is the first study of its kind.

Analysis of the impact of mouse strain on drug exposure is quite limited in the broader scientific literature. Studies often involve a single compound and are not designed to capture critical PK parameters that are used to inform decisions on drug discovery teams. For example, McCarthy et al. (2004) compared the pharmacokinetics of the psychostimulant cocaine in CD-1 and C57BL/6 mice with chronic dosing and reported higher cocaine exposure in C57BL/6 mice compared with CD-1 mice. Guo et al. (2006) evaluated warfarin metabolism in 13 different inbred strains of mice, including BALB/c and C57BL/6, after a single intraperitoneal dose (Guo et al., 2006). The group found substantial differences in formation of certain warfarin metabolites; however, parent warfarin area under the plasma concentration-time curve was within 2.1fold for all strains (Guo et al., 2006). In another study, cotinine and nicotine were independently dosed to groups of DBA/2 and C57BL/6 mice; the resulting PK parameters (area under the curve, $\mathrm{CL} / \mathrm{F}, t_{1 / 2}$, and $C_{\max }$ ) for both strains were within twofold for parent nicotine and cotinine despite seeing a strain-dependent difference in metabolite formation for each compound (Siu and Tyndale, 2007). When taken together, these studies suggest that although certain metabolic pathways may be affected by strain, the elimination of a compound is generally governed by multiple pathways, and as a result, absolute exposure remains relatively consistent between strains.

When selecting compounds in this study to compare PK parameters across mouse strains, we sought to cover as much chemical and ADME space as feasible. We chose compounds with a wide variation in lipophilicity, charge state, chemical functionality, and molecular weight. We also selected compounds that undergo various routes of elimination, including phase I metabolism (CYP1A2, CYP2C8, CYP2C9, CYP2D6, and CYP3A), phase II metabolism (UDP-glucuronosyltransferases), urinary elimination, and biliary elimination in humans. The limitations here are recognized, as the sample set is relatively small, and as such, not all elimination routes are covered. Additionally, the routes of elimination were based on available human data rather than mouse, so we recognize that there may be some interspecies differences. Despite these caveats, the group of compounds used in this study represents a cross section of leads that may arise in drug discovery.

We analyzed the data multiple ways to understand the variability of data between strains. By far the most stringent of metrics was statistical

TABLE 8

Chlorpromazine PK parameters for studies performed on three separate days

\begin{tabular}{|c|c|c|c|c|c|c|}
\hline & & Study 1 & Study 2 & Study 3 & $P$ value & $\% \mathrm{CV}$ \\
\hline \multirow[t]{6}{*}{ CD-1 } & CL (1/h per kilogram) & $4.5 \pm 0.46$ & $5.5 \pm 0.56$ & $4.4 \pm 0.72$ & 0.11 & 13 \\
\hline & $\mathrm{V}_{\mathrm{ss}}(1 / \mathrm{kg})$ & $15 \pm 4.9$ & $13 \pm 2.3$ & $12 \pm 6.2$ & 0.78 & 10 \\
\hline & $t_{1 / 2}(\mathrm{~h})$ & $2.8 \pm 1.1$ & $2.3 \pm 0.48$ & $2.7 \pm 1.5$ & 0.85 & 10 \\
\hline & $C_{\max }(\mu \mathrm{M})$ & $0.17 \pm 0.056$ & $0.12 \pm 0.015$ & $0.20 \pm 0.015$ & 0.075 & 25 \\
\hline & $T_{\max }(\mathrm{h})$ & $0.5(0.5-1)$ & $1(0.5-2)$ & $1(0.5-1)$ & ND & ND \\
\hline & $\mathrm{F}(\%)$ & $17 \pm 1.1$ & $18 \pm 3.2$ & $21 \pm 5.9$ & 0.40 & 11 \\
\hline \multirow[t]{6}{*}{ C57BL/6 } & CL (1/h per kilogram) & $1.7 \pm 0.46$ & $1.6 \pm \mathrm{ND}$ & $2.6 \pm 1.1$ & 0.44 & 29 \\
\hline & $\mathrm{V}_{\mathrm{ss}}(1 / \mathrm{kg})$ & $18 \pm 3.0$ & $21 \pm N D$ & $19 \pm 4.9$ & 0.76 & 9.1 \\
\hline & $t_{1 / 2}(\mathrm{~h})$ & $9.1 \pm 3.4$ & $12 \pm \mathrm{ND}$ & $7.1 \pm 4.3$ & 0.56 & 27 \\
\hline & $C_{\max }(\mu \mathrm{M})$ & $0.082 \pm 0.0072$ & $0.075 \pm 0.012$ & $0.11 \pm 0.025$ & 0.070 & 21 \\
\hline & $T_{\max }(\mathrm{h})$ & $1(1-1)$ & $0.5(0.5-1)$ & $0.5(0.5-1)$ & ND & $\mathrm{ND}$ \\
\hline & $\mathrm{F}(\%)$ & $7.1 \pm 1.1$ & $10 \pm 3.9$ & $10 \pm 2.2$ & 0.38 & 19 \\
\hline
\end{tabular}

$\mathrm{ND}$, not determined 
TABLE 9

Interstrain comparison for hepatocyte $\mathrm{CL}_{\text {int }}$ across three mouse strains and eight compounds

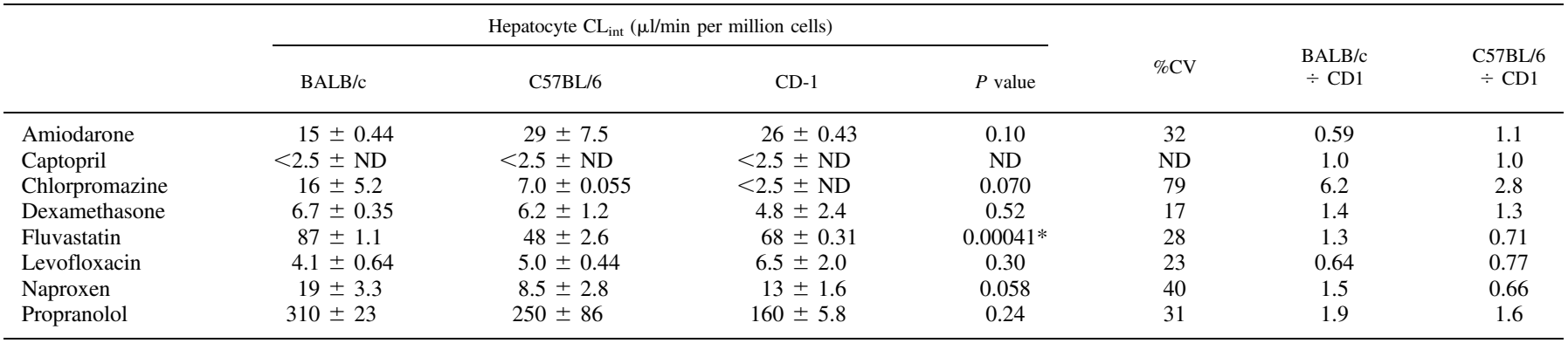

$\mathrm{ND}$, not determined.

${ }^{*} P$ value of 0.05 or less found by a one-way ANOVA test.

analysis using ANOVA. Using this test, we found that there was not a $P$ value less than 0.05 for $50 \%, 75 \%, 63 \%, 63 \%$, and $63 \%$ of compounds in observed $\mathrm{CL}, \mathrm{V}_{\mathrm{ss}}, t_{1 / 2}, C_{\max }$, and $\mathrm{F}$, respectively. Chlorpromazine was an outlier, with strain-dependent differences observed in CL, $\mathrm{V}_{\mathrm{ss}}$, and $t_{1 /}$ 2. Second, we used percent $\mathrm{CV}$ as a metric for the dispersion of parameters. When applying this metric, we found that for $88 \%, 100 \%$, $75 \%, 63 \%, 75 \%$, and $63 \%$ of compounds, the CV fell within $50 \%$ for $\mathrm{CL}, \mathrm{V}_{\mathrm{ss}}, t_{1 / 2}, C_{\max }, T_{\max }$, and $\mathrm{F}$, respectively. Understanding the statistical significance of variability is important; however, given the inherent variability of PK in animals and the limited number of mice used within each study $(n=3)$, basing decisions entirely on statistical approaches seems overly prescriptive. As a more empirical and straightforward approach, we also used a twofold cutoff to look for strain-dependent differences in PK parameters. In our experience, tolerance for PK variability in early discovery, often referred to as screening PK, is generally acceptable if parameters are within twofold (see discussion below). Using this tolerance for variability, we found that parameters were within twofold for $88 \%, 100 \%, 75 \%, 76 \%, 94 \%$, and $88 \%$ of compounds for $\mathrm{CL}, \mathrm{V}_{\mathrm{ss}}, t_{1 / 2}, C_{\max }, T_{\max }$, and $\mathrm{F}$, respectively.

Screening PK experiments are carried out in a relatively highthroughput fashion at relatively low doses, and as a result, there is an expectation for appreciable variability. These experiments are often used to guide the prioritization of molecules, and the values derived from these experiments are treated cautiously rather than absolutely. To assess variability in PK parameters, we ran an intravenous and oral study using chlorpromazine dosed to cohorts $(n=3)$ of CD-1 and C57BL/6 animals on 3 separate days. The data showed little variability; percent $\mathrm{CV}$ was less than $30 \%$ for all parameters measured, and there was no case in which we observed a $P$ value less than 0.05 for ANOVA of PK parameters. Although we generally observed little interday variability in PK parameters for a single compound (chlorpromazine), the scientific community generally accepts twofold variability in PK parameters from in vivo studies (Food and Drug Administration, 2003; Abduljalil et al., 2014; Daublain et al., 2017), acknowledging diversity in subjects (animal or human); variability in drug metabolism enzymes and transporter expression because of genetics, diet, and environmental factors (Yang et al., 2013; Tracy et al., 2016); and possible experimental errors. The Food and Drug Administration, 2003 guidance on Pharmacokinetics in Patients with Impaired Hepatic Function recommends using a confidence interval approach (twofold or greater increase in area under the curve) rather than statistical significance to indicate PK differences in hepatically impaired patients. Primary literature to support a twofold threshold in preclinical species is sparse; however, authors in a recent publication used a twofold cutoff when considering tolerable intra-animal and interanimal screening PK variability (Daublain et al., 2017). In their analysis of a large data set $(\sim 17,000$ compounds) of observed interanimal PK for all typical preclinical animals (mouse, rats, dogs, and monkeys) combined, the authors observed the variability was within twofold for approximately $80 \%$ and $60 \%$ of all intravenous and oral dosing events, respectively (Daublain et al., 2017). These data show that for the same compound, within the same dosing event, the PK variability can be somewhat high; however, it generally falls within twofold. In this study, using a twofold tolerance for variability, we found that parameters were within twofold for $88 \%, 100 \%, 75 \%, 76 \%, 94 \%$, and $88 \%$ of compounds for $\mathrm{Cl}, \mathrm{V}_{\mathrm{ss}}, t_{1 / 2}$, $C_{\max }, T_{\max }$, and F, respectively, supporting the idea that single-strain PK is acceptable for discovery PK screening.

Finally, to see if in vivo strain-dependent differences in CL could be observed in vitro, we measured the hepatocyte $\mathrm{CL}_{\text {int }}$ in each strain. Using CD-1 as the comparator strain, we found $88 \%$ of compounds had hepatocyte $\mathrm{CL}_{\mathrm{int}}$ values that fall within twofold for both $\mathrm{C} 57 \mathrm{BL} / 6$ and BALB/c. Interestingly, the outlier compound was chlorpromazine, which was the compound with the most observed straindependent $\mathrm{CL}$ in vivo. Looking further, we found the rank ordering for chlorpromazine hepatocyte $\mathrm{CL}_{\text {int }}$ (BALB/c $>\mathrm{C} 57 \mathrm{BL} / 6>\mathrm{CD}-1$ ) did not match the $\mathrm{CL}$ in vivo (BALB/c $>\mathrm{CD} 1>\mathrm{C} 57 \mathrm{BL} / 6)$. Based on this, hepatocyte $\mathrm{CL}_{\text {int }}$ did not appear to be predictive of $C L$ differences in vivo, which implies that multiple-strain hepatocyte $\mathrm{CL}_{\text {int }}$ screening may be of little utility on its own without additional data. Perhaps scaling intrinsic hepatocyte clearance to systemic clearance by incorporating strain-specific plasma protein binding, hepatocyte binding, and physiologic scaling factors would provide more utility toward predicting interstrain differences in clearance. This exercise is a subject of future work.

Overall, the in vitro results in this study were consistent with previous work. Richmond et al. (2010) measured microsomal clearance for 96 model compounds using liver microsomes obtained from BALB/c, C57BL/6, and CD-1 mice. The clearance values were binned into either low, medium, or high clearance categories, and the authors found that approximately $95 \%$ of compounds had no strain-dependent differences in the categorical interpretation of clearance (Richmond et al., 2010). Löfgren et al. (2004) also showed that enzyme activity between CD-1 and C57BL/6 microsomes was within twofold for 12 of 13 cytochrome P450 probe substrates tested. Similarly, the authors from another study found a lack of strain dependence of coumarin 7-hydroxylase activity in microsomes prepared from eight different strains of mouse, including BALB/c and C57BL/6 (van Iersel et al., 1994). Although it appears the clearance is largely unaffected by strain for most compounds, it should be noted that specific examples of 
strain-dependent differences have been shown in enzyme kinetics, metabolite formation, enzyme abundance, and enzyme inducibility (Siu and Tyndale, 2007; Wang et al., 2019).

In summary, we measured PK for eight small molecules in three strains of mice. Some statistical strain-dependent differences were observed; however, we found good general agreement of PK between strains, as $88 \%, 100 \%, 75 \%, 76 \%, 94 \%$, and $88 \%$ of compounds were within twofold across strains for $\mathrm{CL}, \mathrm{V}_{\mathrm{ss}}, t_{1 / 2}, C_{\max }, T_{\max }$, and $\mathrm{F}$, respectively. Overall, we recommend that an approach using a single strain of mouse is appropriate for discovery screening PK if caution is exercised. The data suggest that testing hepatocyte $\mathrm{CL}_{\text {int }}$ across strains may not be predictive of in vivo CL on its own. Therefore, in cases in which the PK strain does not match the PD or Tox strain, we recommend to periodically spot check major strain-dependent differences within a chemical series to mitigate risk of PK/PD or PK-Tox translational errors.

\section{Authorship Contributions}

Participated in research design: Barr, Rock, Wahlstrom, Dahal.

Conducted experiments: Tran.

Performed data analysis: Barr, Tran, Dahal.

Wrote or contributed to the writing of the manuscript: Barr, Rock, Wahlstrom, Dahal.

\section{References}

Abduljalil K, Cain T, Humphries H, and Rostami-Hodjegan A (2014) Deciding on success criteria for predictability of pharmacokinetic parameters from in vitro studies: an analysis based on in vivo observations. Drug Metab Dispos 42:1478-1484.
Barr JT, Lade JM, Tran TB, and Dahal UP (2019) Fraction unbound for liver microsome and hepatocyte incubations for all major species can be approximated using a single-species surrogate. Drug Metab Dispos 47:419-423.

Daublain P, Feng KI, Altman MD, Martin I, Mukherjee S, Nofsinger R, Northrup AB, TschirretGuth R, Cartwright M, and McGregor C (2017) Analyzing the potential root causes of variability of pharmacokinetics in preclinical species. Mol Pharm 14:1634-1645.

Food and Drug Administration (2003) Available from:, U.S. Food and Drug AdministrationCenter for Drug Evaluation and Research, Silver Spring, MD.

Guo Y, Weller P, Farrell E, Cheung P, Fitch B, Clark D, Wu SY, Wang J, Liao G, Zhang Z, et al. (2006) In silico pharmacogenetics of warfarin metabolism. Nat Biotechnol 24:531-536.

Löfgren S, Hagbjörk AL, Ekman S, Fransson-Steen R, and Terelius Y (2004) Metabolism of human cytochrome P450 marker substrates in mouse: a strain and gender comparison. Xenobiotica 34:811-834.

MacCallum DM and Odds FC (2002) Influence of grapefruit juice on itraconazole plasma levels in mice and Guinea pigs. J Antimicrob Chemother 50:219-224.

McCarthy LE, Mannelli P, Niculescu M, Gingrich K, Unterwald EM, and Ehrlich ME (2004) The distribution of cocaine in mice differs by age and strain. Neurotoxicol Teratol 26:839-848.

Richmond W, Wogan M, Isbell J, and Gordon WP (2010) Interstrain differences of in vitro metabolic stability and impact on early drug discovery. J Pharm Sci 99:4463-4468.

Siu EC and Tyndale RF (2007) Characterization and comparison of nicotine and cotinine metabolism in vitro and in vivo in DBA/2 and C57BL/6 mice. Mol Pharmacol 71:826-834.

Tracy TS, Chaudhry AS, Prasad B, Thummel KE, Schuetz EG, Zhong XB, Tien YC, Jeong H, Pan $\mathrm{X}$, Shireman LM, et al. (2016) Interindividual variability in cytochrome P450-mediated drug metabolism. Drug Metab Dispos 44:343-351.

van Iersel M, Walters DG, Price RJ, Lovell DP, and Lake BG (1994) Sex and strain differences in mouse hepatic microsomal coumarin 7-hydroxylase activity. Food Chem Toxicol 32:387-390.

Wang S, Chen L, Wang Q, He Z, Chen S, Zhang H, Li H, Guo P, Li Q, Zhang R, et al. (2019) Strain differences between CD-1 and C57BL/6 mice in expression of metabolic enzymes and DNA methylation modifications of the primary hepatocytes. Toxicology 412:19-28.

Yang L, Price ET, Chang CW, Li Y, Huang Y, Guo LW, Guo Y, Kaput J, Shi L, and Ning B (2013) Gene expression variability in human hepatic drug metabolizing enzymes and transporters. PLoS One 8:e60368.

Address correspondence to: Upendra P. Dahal, Pharmacokinetics and Drug Metabolism, Amgen Inc., 1120 Veteran's Blvd., South San Francisco, CA 94080. E-mail: udahal@amgen.com 